\title{
A Game-Theoretical Model Predictive Control Framework for Electricity Markets
}

\author{
Aswin Kannan and Victor M. Zavala
}

\begin{abstract}
We present a game-theoretical model predictive control (MPC) framework for competitive electricity markets. We demonstrate that an MPC construct can be used to systematically analyze the effects of ramp constraints, initial conditions, dynamic disturbances, forecast horizon length, market manipulation, and bidding frequency on market performance. We illustrate the capabilities of the framework using a numerical case study.
\end{abstract}

\section{NOMENCLATURE}

$\underset{\mathcal{S}}{S}$
$\mathcal{C}$
$\mathcal{T}$
Variables
$q_{t}^{i}$
$\Delta q_{t}^{i}$
$p_{t}$
$\lambda_{t}^{i}$
$\underline{\pi}_{t}^{i}, \bar{\pi}_{t}^{i}$
$\underline{\nu}_{t}^{i}, \bar{\nu}_{t}^{i}$

Set of suppliers

Set of consumers

Set of time steps

$\underset{S}{\text { Constants }}$
$C$
$T$
$d_{t}^{j}$
$h^{i}, g^{i}$
$\underline{r}^{i}, \bar{r}^{i}$
$\underline{q}_{\bar{q}}^{i}, \bar{q}^{i}$

Production quantities (MW)

Incremental production quantities (MW)

Price ( $\$$ MW)

Adjoints for dynamic system (\$/MW)

Multipliers production (\$/MW)

Multipliers ramps $(\$ / \mathrm{MW})$

Number of suppliers

Number of consumers

Number of time steps in horizon

Demand (MW)

Coefficients cost function

(\$/MW,\$/MW ${ }^{2}$ )

Bounds ramps (\$/MW)

Bounds production quantities (\$/MW)

Initial conditions suppliers (MW)

\section{INTRODUCTION}

Diverse market models have been proposed in the literature to analyze the impact of different dynamic disturbances (e.g., weather, load, fuel prices, and wind supply), physical constraints (e.g., transmission congestion), and gaming behaviors (e.g., bidding strategies) on market performance and prices [23]. These models range from data-based time-series models [20], [10] to mechanistic models based on agent-based systems [8], [22] and game-theoretical formulations [6], [16].

Game-theoretical models can be used to establish market properties in a systematic manner and thus provide more comprehensive predictive capabilities. Several models based on a range of market structure assumptions have been proposed, all of which are static in the sense that they assume some sort of steady-state behavior of the fundamental market drivers. These models can provide a reasonable representation of the market under stationarity or strong periodicity of dynamic disturbances. Consequently, they can be used to analyze longterm behavior and physical constraints such as transmission congestion in planning and market design exercises. However, static models are not capable of explaining the effect of other dynamic constraints and non-stationary behavior, which is the most common case in real-time operations. Consequently, their use in market monitoring and price forecasting is limited.

A widely used dynamic market model originally proposed in [1], [2] assumes that the players bid recursively in the direction that minimizes their marginal cost. Every bid can be interpreted as a steepest-descent step that converges to a steady-state equilibrium. While this model is useful for analyzing static (instantaneous) market stability properties, it is based on mathematical rather than mechanistic assumptions and thus has limited applicability. Recently, a dynamic market model based on model predictive control (MPC) concepts was proposed in [15], [14]. Here, supply functions and forecast horizon concepts are incorporated into the model. These concepts provide a more natural representation of actual bidding procedures. This model has been used to analyze the effect of wind supply variability on prices under high penetration levels. A limitation of this framework, however, is that the dynamic model of the players is still based on the marginal-cost descent assumption.

The main observation motivating this work is the fact that the effects of physical dynamic constraints and market design specifications such as bidding frequency and forecast horizon can be analyzed systematically using MPC concepts. In particular, ramping constraints restrict bidding procedures at subsequent time intervals (day-ahead and real-time markets) and thus affect short-term and long-term dynamic market stability and performance. In some sense, ramping constraints affect market performance much as transmission congestion does [12]. The key difference, however, is that the effect of ramping constraints propagates forward in time while the effects of transmission congestion are static (instantaneous).

The effects of manipulation of ramp constraints on market behavior was studied in [19]. Ramp rates represent the maximum change that a generator can achieve in their power output level within a given time interval [25]. They implicitly represent the time that it will take the generator control system to move the power output level from the current level to the desired target. These ramp rates depend on multiple physical factors such as controller performance [3], [5], thermal stresses, and wall capacitances [21]. Large generators such as those running on coal and nuclear fuel are operated at base load and are not ramped. Smaller generators and combinedcycle plants running on natural gas provide ramping capacity 
and are used to regulate real-time deviations of the loads from forecasts. The generation costs of these ramping units are much higher than those of base units due to higher prices of natural gas and capital costs. The effect of ramping units on electricity prices will become stronger in the presence of more volatile and dynamic environments, such as those expected under high wind-supply penetration and smart-grid programs.

We propose a game-theoretical MPC framework that uses a closer physical representation of generator dynamic constraints and bidding procedures. We demonstrate that the framework can be used to analyze systematically the effects of ramping limits, initial conditions, intermittent supply, forecast horizons, market manipulation, and bidding frequency on market performance. We provide numerical results under several operational scenarios in order to illustrate the consistency and analytical capabilities of the model.

The paper is structured as follows: In the following section we present the basic model, discuss underlying assumptions, and offer a solution strategy. In Section III we discuss model predictive control implementation details necessary to simulate day-ahead markets and evaluate market performance. In Section IV we present numerical results. The last section provides concluding remarks and directions for future work.

\section{Game-Theoretical Dynamic Model}

In this section, we present a basic dynamic game-theoretical model. The model is targeted to capture dynamic effects on market performance. Consequently, simplifications have been made to avoid unnecessary complexity. Potential extensions are discussed later on as part of future work.

We consider a unilateral Nash-Cournot market model where the suppliers bid production quantities (power levels) to maximize their profit and a central entity such as the Independent System Operator (ISO) that clears the market by balancing supply and demand. The consumer demands are assumed to be fixed. Each supplier $i \in \mathcal{S}=\{1 . . S\}$ is solve sproblem (1).

$$
\begin{aligned}
\max _{q_{t}^{i}} & \sum_{t \in \mathcal{T}}\left(p_{t} q_{t}^{i}-c_{t}^{i}\left(q_{t}^{i}\right)\right) \\
\text { s.t. } & q_{t+1}^{i}-q_{t}^{i} \leq \bar{r}^{i}, t \in \mathcal{T}^{-} \\
& q_{t}^{i}-q_{t+1}^{i} \leq \underline{r}^{i}, t \in \mathcal{T}^{-} \\
& \underline{q}^{i} \leq q_{t}^{i} \leq \bar{q}^{i}, t \in \mathcal{T} \\
& q_{0}^{i}=\bar{q}_{0}^{i} .
\end{aligned}
$$

Here $T$ is the forecast horizon and $\mathcal{T}:=\{0 . . T\}$ is the set of time steps. We also define the set $\mathcal{T}^{-}:=\mathcal{T} \backslash\{T\}$. Symbols $\underline{r}^{i}$ and $\bar{r}^{i}$ denote the down and up ramp rates. The bidding production quantities $q_{t}^{i}$ are bounded by the down and up limits $\underline{q}_{t}^{i}$ and $\bar{q}_{t}^{i}$, respectively. The initial conditions for the production quantities are given by $\bar{q}_{0}^{i}$ and are fixed. These represent the current power output levels of the generators.

The cost function, defined by $c_{t}^{i}(\cdot)$, is assumed to be any convex function (e.g., linear, quadratic, or piece-wise linear).
Here, we consider quadratic costs of the form

$$
c_{t}^{i}\left(q_{t}^{i}\right)=h^{i} \cdot q_{t}^{i}+\frac{1}{2} g^{i} \cdot\left(q_{t}^{i}\right)^{2} .
$$

The price is defined by $p_{t}$ and is given by by the market clearing condition

$$
\sum_{i \in \mathcal{S}} q_{t}^{j}=\sum_{j \in \mathcal{C}} d_{t}^{j}
$$

where $d_{t}^{j}$ are the consumer demands $j \in \mathcal{C}=\{1 . . C\}$. The market clearing condition can be interpreted as the minimization of the imbalance of supply and demand. Consequently, the prices $p_{t}$ can be seen as the Lagrange multiplier of the clearing conditions.

We note that the game can be posed as a discrete-time dynamic game in state-space form as:

$$
\begin{gathered}
\sum_{i \in \mathcal{S}} q_{t}^{i}=\sum_{j \in \mathcal{C}} d_{t}^{j}, t \in \mathcal{T} \\
i \in \mathcal{S}\left\{\max _{\Delta q_{t}^{i}} \sum_{t \in \mathcal{T}}\left(p_{t} q_{t}^{i}-c_{t}^{i}\left(q_{t}^{i}\right)\right)\right. \\
\text { s.t. } q_{t+1}^{i}=q_{t}^{i}+\Delta q_{t}^{i}, t \in \mathcal{T}^{-} \\
\underline{r}^{i} \leq \Delta q_{t}^{i} \leq \bar{r}^{i}, t \in \mathcal{T}^{-} \\
\underline{q}^{i} \leq q_{t}^{i} \leq \bar{q}^{i}, t \in \mathcal{T} \\
q_{0}^{i}=\bar{q}_{0}^{i} .
\end{gathered}
$$

This representation is typical in the dynamic games literature [7]. The state-space representation enables us to analyze the market as a game-theoretical MPC problem. Here, the production quantities $q_{t}^{i}$ can be interpreted as differential states coupled in time, and the increments $\Delta q_{t}^{i}$ can be interpreted as the controls. The price acts as an algebraic state since it is not directly coupled in time. The initial conditions play a critical role on the overall market performance since they propagate the effects of ramping constraints in time. In addition we note that, in the presence of ramping constraints, the feasible set of the game depends on the initial conditions. For instance, the demand at future times might not be reachable for a given set of initial conditions and ramping limits. The solution of the game (4) gives equilibrium trajectories, or a dynamic equilibrium, for the supply quantities and prices that satisfy the demands at each point in time and maximize social welfare. Note that the trajectories depends on the initial conditions, forecast horizon, and on the ramp rates of the generators.

We note that the Nash-Cournot formulation proposed here gives the same solution as if the suppliers do not take into account ramping constraints and these are handled centrally by the ISO. Our objective is to understand how different dynamic factors affect the performance of the market. Because of this, we do not consider more sophisticated market formulations including, among others, transmission constraints and supply functions. While these formulations will give more realistic predictive capabilities to the model, they do not provide extra information into the basic concepts analyzed here. 
We can solve the market problem by solving the associated complementarity system [16]. We define the Lagrange function for supplier $i$ as follows:

$$
\begin{aligned}
\mathcal{L}^{i}= & \sum_{t \in \mathcal{T}}-\left(p_{t} q_{t}^{i}-c_{t}^{i}\left(q_{t}^{i}\right)\right)+\sum_{t \in \mathcal{T}^{-}} \lambda_{t+1}^{i}\left(q_{t+1}^{i}-q_{t}^{i}-\Delta q_{t}^{i}\right) \\
& +\lambda_{0}^{i}\left(q_{0}^{i}-\bar{q}_{0}^{i}\right) \\
& -\sum_{t \in \mathcal{T}^{-}} \underline{\nu}_{t}^{i}\left(\Delta q_{t}^{i}-\underline{r}^{i}\right)-\sum_{t \in \mathcal{T}^{-}} \bar{\nu}_{t}^{i}\left(\bar{r}^{i}-\Delta q_{t}^{i}\right) \\
& -\sum_{t \in \mathcal{T}} \underline{\pi}_{t}^{i}\left(q_{t}^{i}-\underline{q}^{i}\right)-\sum_{t \in \mathcal{T}} \bar{\pi}_{t}^{i}\left(\bar{q}^{i}-q_{t}^{i}\right), i \in \mathcal{S}
\end{aligned}
$$

Here, $\lambda_{t}^{i}$ are the Lagrange multipliers for the dynamic system (adjoints) and $\underline{\nu}_{t}^{i}, \bar{\nu}_{t}^{i}, \underline{\pi}_{t}^{i}$ and $\bar{\pi}_{t}^{i}$ are the bound multipliers. This gives the following mixed linear complementarity system:

$$
\begin{aligned}
& \sum_{i \in \mathcal{S}} q_{t}^{i}-\sum_{j \in \mathcal{C}} d_{t}^{j}=0, t \in \mathcal{T} \\
& \nabla_{q_{t}^{i}} \mathcal{L}=0, t \in \mathcal{T} \\
& \nabla_{\Delta q_{t}^{i}} \mathcal{L}=0, t \in \mathcal{T}^{-} \\
& \lambda_{t+1}^{i} \perp q_{t+1}^{i}-\left(q_{t}^{i}+\Delta q_{t}^{i}\right)=0, t \in \mathcal{T}^{-} \\
& 0 \leq \underline{\nu}_{t}^{i} \perp\left(\Delta q_{t}^{i}-\underline{r}^{i}\right) \geq 0, t \in \mathcal{T}^{-} \\
& 0 \leq \bar{\nu}_{t}^{i} \perp\left(\bar{r}^{i}-\Delta q_{t}^{i}\right) \geq 0, t \in \mathcal{T}^{-} \\
& 0 \leq \underline{\pi}_{t}^{i} \perp\left(q_{t}^{i}-q^{i}\right) \geq 0, t \in \mathcal{T} \\
& 0 \leq \bar{\pi}_{t}^{i} \perp\left(\bar{q}^{i}-\bar{q}_{t}^{i}\right) \geq 0, t \in \mathcal{T} \\
& \lambda_{0}^{i} \perp\left(q_{0}^{i}-\bar{q}_{0}^{i}\right)=0
\end{aligned}
$$

where

$$
\begin{gathered}
\nabla_{q_{t}^{i}} \mathcal{L}^{i}=-p_{t}+\frac{\partial c_{t}^{i}}{\partial q_{t}^{i}}+\lambda_{t}^{i}-\lambda_{t+1}^{i}-\underline{\pi}_{t}^{i}+\bar{\pi}_{t}^{i}=0, \\
t \in \mathcal{T}^{-} \\
\nabla_{q_{T}^{i}} \mathcal{L}^{i}=-p_{T}+\frac{\partial c_{T}^{i}}{\partial q_{T}^{i}}+\lambda_{T}^{i}-\underline{\pi}_{T}^{i}+\bar{\pi}_{T}^{i}=0 \\
\nabla_{\Delta q_{t}^{i}} \mathcal{L}^{i}=-\lambda_{t+1}^{i}-\underline{\nu}_{t}^{i}+\bar{\nu}_{t}^{i}, t \in \mathcal{T}^{-},
\end{gathered}
$$

for $i \in \mathcal{S}$. Note that the optimality conditions for the ISO's problem are the market clearing conditions (4a).

The multipliers or adjoints $\lambda_{t}^{i}$ of the dynamic constraint (4c) can be interpreted as ramping prices, analogous to the congestion prices arising from transmission constraints. These multipliers reflect the sensitivity of the production costs to the ramp rates. If the generator is not ramp-constrained, the multiplier will be zero. If the generator is constrained, the magnitude of the multiplier will be given by the generation cost. From (7) we can note that the ramping prices are coupled in time and propagate backwards.

The resulting complementarity systems can be extremely large, depending on the length of the prediction horizon and the number of players. To solve these systems, one can use complementarity solvers such as PATH [11] or general nonlinear optimization solvers such as KNITRO [9] and IPOPT [24]. For the problems considered in this work we have found that a nonlinear optimization approach coupled to a $\ell_{1}$ penalty formulation is numerically more robust. In this approach, the objective is to minimize the complementarity products [18], [13], [4]. For (6), the problem takes the form:

$$
\left.\begin{array}{rl}
\min & \sum_{i \in \mathcal{S}} \sum_{t \in \mathcal{T}^{-}}\left(\underline{\nu}_{t}^{i}\left(\Delta q_{t}^{i}-\underline{r}^{i}\right)+\bar{\nu}_{t}^{i}\left(\bar{r}^{i}-\Delta q_{t}^{i}\right)\right) \\
& +\sum_{i \in \mathcal{S}} \sum_{t \in \mathcal{T}}\left(\underline{\pi}_{t}^{i}\left(q_{t}^{i}-\underline{q}^{i}\right)+\bar{\pi}_{t}^{i}\left(\bar{q}^{i}-q_{t}^{i}\right)\right) \\
\text { s.t. } & \sum_{i \in \mathcal{S}} q_{t}^{i}-\sum_{j \in \mathcal{C}} d_{t}^{j}=0, t \in \mathcal{T} \\
& \nabla_{q_{t}^{i}} \mathcal{L}=0, t \in \mathcal{T} \\
& \nabla_{\Delta q_{t}^{i}} \mathcal{L}=0, t \in \mathcal{T}^{-} \\
& q_{t+1}^{i}-\left(q_{t}^{i}+\Delta q_{t}^{i}\right)=0, t \in \mathcal{T}^{-} \\
& 0 \leq \underline{\nu}_{t}^{i},\left(\Delta q_{t}^{i}-\underline{r}^{i}\right) \geq 0, t \in \mathcal{T}^{-} \\
& 0 \leq \bar{\nu}_{t}^{i},\left(\bar{r}^{i}-\Delta q_{t}^{i}\right) \geq 0, t \in \mathcal{T}^{-} \\
& 0 \leq \underline{\pi}_{t}^{i},\left(q_{t}^{i}-q^{i}\right) \geq 0, t \in \mathcal{T} \\
& 0 \leq \bar{\pi}_{t}^{i},\left(\bar{q}^{i}-\bar{q}_{t}^{i}\right) \geq 0, t \in \mathcal{T} \\
& \left(q_{0}^{i}-\bar{q}_{0}^{i}\right)=0
\end{array}\right\} i \in \mathcal{S} .
$$

\section{Model Predictive Control}

To analyze real-time market operations, one can solve the dynamic game problem in a receding-horizon manner with a predefined forecast horizon $T$. The bidding frequency is implicitly given by the sampling time. This can be used to account for changing conditions of dynamic disturbances such as weather, demands, forecast errors, and fuel prices.

The infinite horizon bidding game $(T=\infty)$ gives the optimal equilibrium trajectory for a given disturbance forecast trajectory. For implementation, however, a receding-horizon bidding strategy is required to avoid computational limitations. The idea is to define a finite horizon $T$ to compute an equilibrium trajectory and to carry out the state to the next window. Typically, the day-ahead market is cleared with a forecast horizon of 24 to 36 hours. Our strategy starts at a given bidding time $t_{\ell}$ with initial conditions $q_{t_{\ell}}^{i}$ and to use these as initial conditions $\bar{q}_{0}^{i}=q_{\ell}^{i}$ to compute the dynamic equilibrium trajectory over horizon $\mathcal{T}=\left\{t_{\ell}, \ldots, t_{\ell}+T\right\}$. The initial conditions are then updated to the generator states at the end of the horizon $\bar{q}_{0}^{i}=q_{t_{\ell}+T}^{i}$. In other words, the horizon is shifted forward by $T$ steps so that the new bidding horizon is $\mathcal{T}=\left\{t_{\ell}+T, \ldots, t_{\ell}+2 T\right\}$. We assume a deterministic setting using perfect forecast in the day-ahead market. With this, it is not necessary to model the real-time market, which takes care of high-frequency load imbalances resulting from day-ahead forecast errors.

The moving horizon policy provides an approximation to the infinite horizon policy which provides the ideal performance. One of the interesting questions that arise in this context is how long the horizon should be?. This is often problem dependent since it depends on the structure of the infinite horizon equilibrium policy. We also note that, in the absence of ramp constraints, the moving and infinite horizon policies coincide since the states $q_{t}^{i}$ are no longer coupled in time. This holds even in the presence of other steady-state constraints such as transmission constraints, cost functions, and bidding strategies such as supply functions. On the other hand, we note that, in the presence of ramping constraints, any errors 
introduced by finite horizon approximations and forecast errors propagate forward in time through the generator states. This is one of the main reasons why the prediction horizon should be as long as possible in order to foresee the future effects of ramping constraints.

\section{NuMERICAL RESUlTS}

In this section, we report on numerical simulations that we conducted under several operational scenarios. Our objective is to illustrate the effect of dynamic constraints on the price dynamics and to demonstrate the consistency of the MPC framework. In addition, we discuss the limitations of recedinghorizon market designs in the presence of strong variations of load and wind supply.

We consider a system with three suppliers and one demand. One of the suppliers has fast dynamics (high ramping capacity) but high cost such as natural gas generators, the second one has slow dynamics but also low cost, and the third one is used as a slack generator with infinite ramp limits (equal to generation capacity) and a large cost. This last supplier acts as a slack to avoid infeasibility. The nominal parameters used are $q=[0,0,0], \bar{q}=[50,70,120], \underline{r}=-[5,10,120], \bar{r}=$ $[5,1 \overline{0}, 120], h=[4,2,5]$, and $g=[2,1,5]$. The ramps were varied in some experiments from their nominal values. We used $\bar{q}_{0}=[0,40,40]$ as initial conditions.
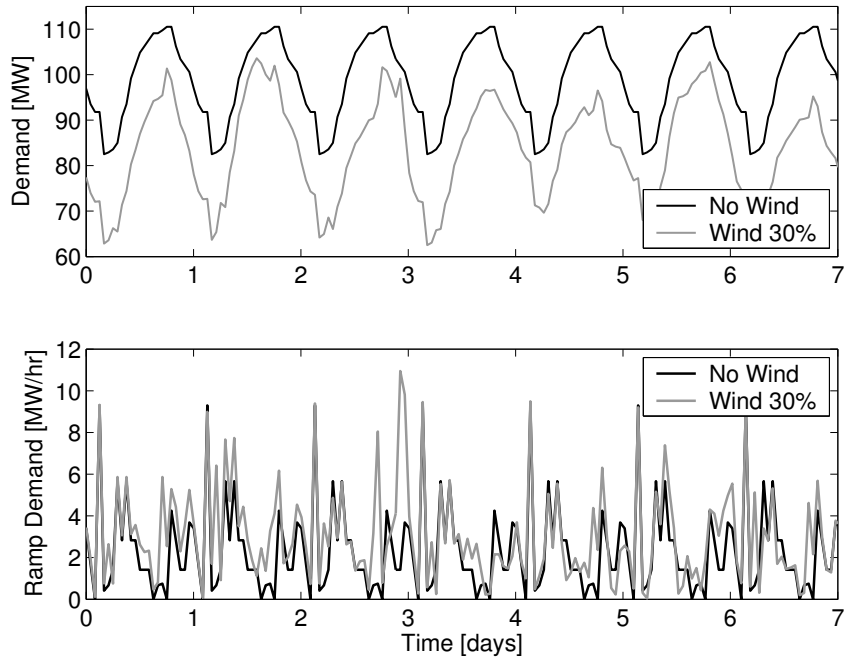

Fig. 1. Total (top) and ramp (bottom) demand for market system over 14 days of operation with $0 \%$ and $30 \%$ wind penetration.

We consider the demand profiles for two cases, one with periodic demand (labeled as "No Wind") and one where the periodic demand is shifted by a wind power profile representing $30 \%$ of wind penetration (labeled as " $30 \%$ Wind"). In the top graph of Figure 1 we present total demands for the two cases. The periodic demand fluctuates between $80 \mathrm{MW}$ and $110 \mathrm{MW}$. In the wind case, the net demand is shifted down (demand minus wind supply) but becomes more volatile. In particular, periodicity is partially destroyed.

In the bottom graph we present the ramp demands for the two demand cases. These have been obtained by computing the absolute differences $\left|d_{t+1}-d_{t}\right|, t \geq 0$. Note that even if the net demand is lower in the wind case, the ramp demands can increase significantly at particular points in time. This situation can be observed clearly at the beginning of the third day. This illustrates how ramping constraints can become more significant under more volatile environments.

\section{A. Effect of Ramp Rates}

We first analyze the effect of ramping constraints. For this analysis, we consider the case with periodic demand. In Figure 2 we present the dynamic equilibria for three ramp scenarios. The first scenario, "High Ramp", corresponds to the nominal ramp values, scenario "Low Ramp" corresponds to a $50 \%$ decrease in the nominal values of the suppliers, and "Infinite Ramp" corresponds to an unconstrained ramp case (ramps set to large value). Note that in the constrained cases the price signals reach a periodic steady-state after a couple of days. In the absence of ramp constraints, the periodic steady-state is reached immediately. Note also that the shape of the steadystate equilibrium is affected by the ramp rates. In particular, the prices are more volatile during high and low peaks when the ramps are lower. In the ramp-constrained case the prices fluctuate between 100 \$/MW and 40 \$/MW in a single day. In the unconstrained case, the prices fluctuate between $80 \$ / \mathrm{MW}$ and $65 \$ \mathrm{MW}$. In Figure 3 we can observe that the bidding

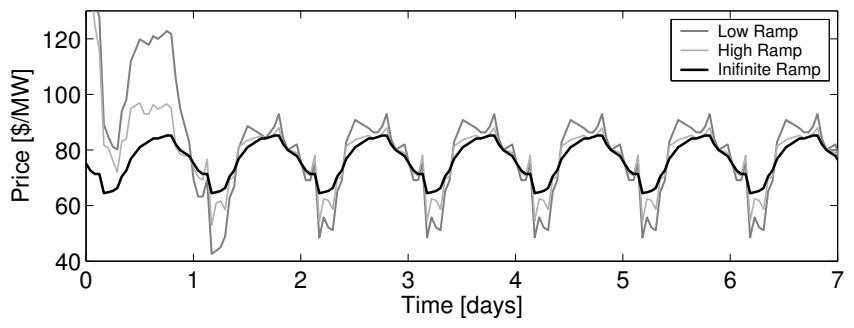

Fig. 2. Effect of ramp limits on price dynamics.

quantities saturate while reaching the periodic steady-state (dashed lines).

In Figure 4 we plot the adjoint variables $\lambda_{t}^{i}$ for the three suppliers. These multipliers reflect the sensitivity of the profit for each supplier to changes in the ramp rates. Note that the multipliers reach a steady-state after seven days and that the greater sensitivity is observed during peaking times, as expected. Supplier 2 is clearly the most sensitive since it has more limited ramping capacity. The adjoint of the slack supplier is zero since the ramps are never active. We have observed that the adjoints tend to diverge for extremely long horizons, introducing numerical problems in the solution. Divergence is attributed mainly to the lack of a terminal constraint in the suppliers problems. We have found that penalizing the profit in the last time step $T$ by a large value stabilizes the adjoints.

\section{B. Effect of Dynamic Disturbances}

In Figure 5 we present the effect of ramps for the case of $30 \%$ wind power penetration. It is clear that the volatility of the 


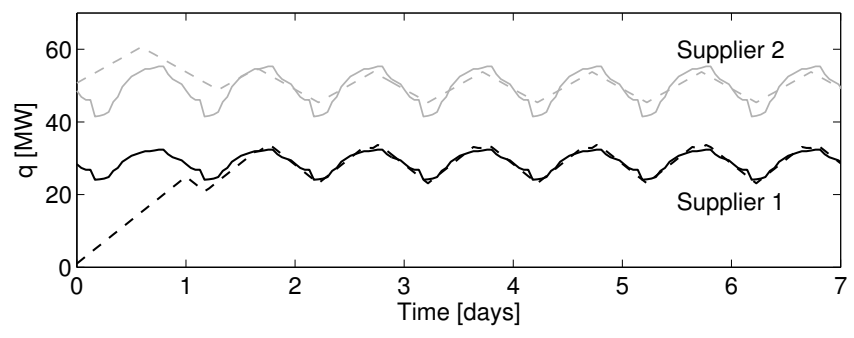

Fig. 3. Effect of ramp limits on bidding dynamics. Solid lines are profiles without ramp limits, and dashed lines are profiles with ramp limits.

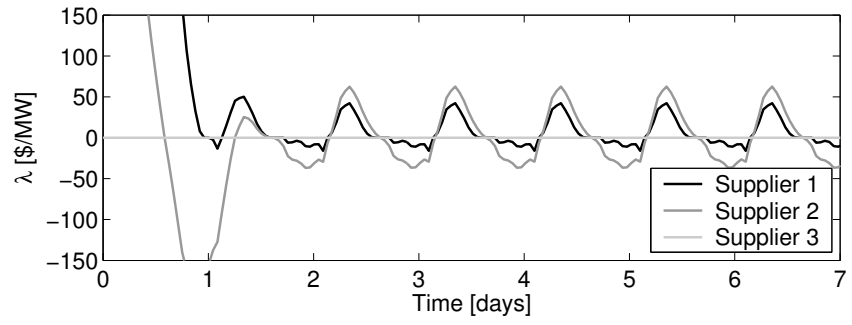

Fig. 4. Adjoint dynamics for suppliers in the presence of ramp limits.

prices increases significantly as the ramp constraints become tighter. In the presence of ramping constraints we observe that wind supply introduces strong price variations. The price ranges from $150 \$ / \mathrm{MW}$ to $40 \$ / \mathrm{MW}$ in a single day. In the unconstrained case (infinite ramps), the prices are significantly more stable and ranges from $60 \$ / \mathrm{MW}$ to $40 \$ / \mathrm{MW}$ in a single day. In addition, we note that the price periodicity is stronger in the absence of ramp constraints. This reflects a decreased sensitivity to wind supply variations. This illustrates that, while the wind supply cost might be very low, the increasing ramp demands can increase price volatility significantly because of the need of additional ramping capacity (natural gas).

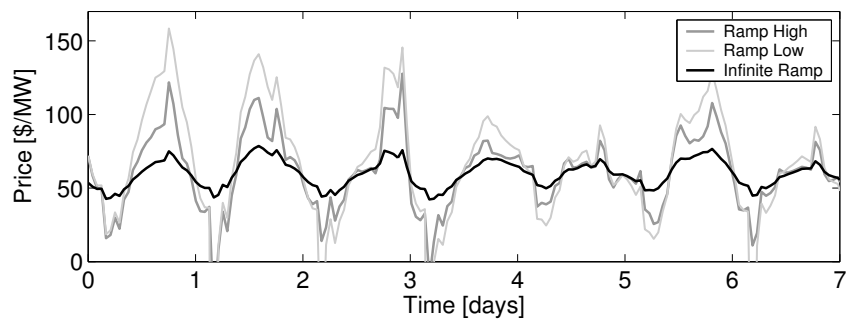

Fig. 5. Effect of ramp limits on bidding dynamics under $30 \%$ wind penetration case.

\section{Effect of Forecast Horizon}

In Figure 6 we illustrate the effect of the forecast horizon on the price signals. We compare the infinite horizon case and the one day-ahead forecast. Note that suboptimality is introduced during periods of strong dynamic variations. In the third day, the prices of the day-ahead case reach 120\$/MW while the optimal ones are around 100\$/MW. We have found that increasing the horizon to two days approximates well the infinite horizon policy. We have also found that, in the case of a perfectly periodic demand, the one day and infinite horizon policies are the same. A critical conclusion from this dynamic analysis is that short horizons only work well under strong stationarity (periodicity) of the load. In the presence of strong dynamic disturbances (wind supply and weather fronts), the horizon should be increased in order to keep prices more stable. This can be explained from the fact that short horizons tend to position generators at suboptimal production levels at the end of the horizon from where subsequent demand profiles not considered in the horizon might not be reachable or can only be reached at a high cost.

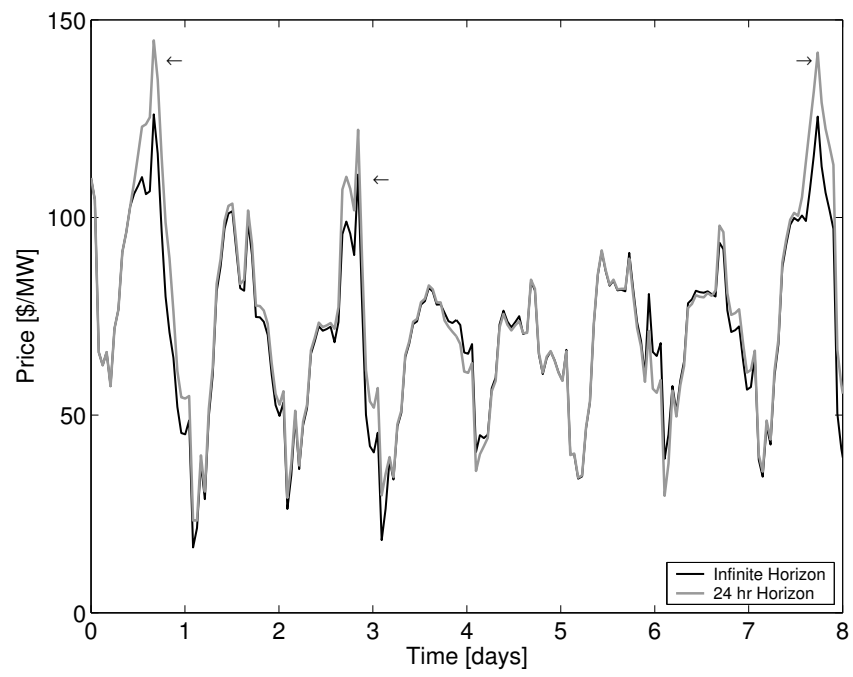

Fig. 6. Effect of horizon length on market performance under $30 \%$ wind penetration case.

\section{Effect of Market Manipulation}

One of the main applications of game theoretical models is the analysis of non-gaming behavior (e.g., market manipulation). In Figure 7, we present the price signals for a perfect game and for the case in which supplier 1 bids randomly (not trying to maximize its profit). From the profiles, it is clear that prices tend to become higher and more volatile in the presence of non-gaming behavior. We note that suboptimal player bids introduce noise to the optimal gaming policy obtained from the market model and raises prices from $\$ 85 / \mathrm{MW}$ to 100 \$/MW. Having the MPC framework, the noise can be identified by solving a state estimation problem. This capability can be used to monitor the market in real-time and to forecast price signals. Another potential use of an MPC framework is the design of market clearing procedures that are less sensitive to noise and manipulation.

\section{Conclusions And Future Work}

We have presented a game-theoretical model predictive control (MPC) framework for electricity markets. We demonstrate that the MPC construct enables a systematic analysis of the effects of ramp constraints, initial conditions, and forecast 


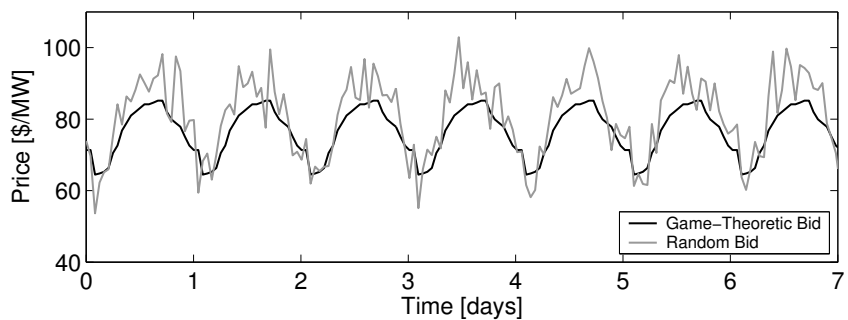

Fig. 7. Price signals under game-theoretic and random bids for supplier 1.

horizons on market performance and price volatility. The framework also enables the analysis of market manipulation (non-optimal bidding) in the form of noise in the prices signals. We have presented numerical experiments to illustrate the consistency and analytical capabilities of the MPC framework.

The proposed framework can be extended in a number of ways to consider more detailed physical effects and market design structures such as transmission constraints and coupled day-ahead and real-time markets (two-settlement markets) [26], [17]. In addition, the MPC construct opens the door to systematic stability analyzes that can be used to better design market structures.

\section{ACKNOWLEDGMENTS}

This work was supported by the U.S. Department of Energy, under Contract No. DE-AC02-06CH11357.

\section{REFERENCES}

[1] F. Alvarado. The stability of power system markets. IEEE Transactions on Power Systems, 14:505-511, 1999.

[2] F. Alvarado. Stability analysis of interconnected power systems coupled with market dynamics. IEEE Transactions on Power Systems, 16:695701,2001

[3] F. D' Amato. Industrial application of a model predictive control solution for power plant startups. IEEE International Conference on Control Applications, pages 243-248, 2006.

[4] M. Anitescu. On solving mathematical programs with complementarity constraints as nonlinear programs. SIAM J. Optim., 15(4):1203-1236, 2005.

[5] C. Aurora, L. Magni, R. Scattolini, P. Colombo, F. Pretolani, and G. Villa. Predictive control of thermal power plants. International Journal of Robust and Nonlinear Control, 14:415-433, 2004.

[6] R. Baldick and W. Hogan. Capacity constrained supply function equilibrium models of electricity markets: Stability, nondecreasing constraints, and function space iterations. In University of California Energy Institute, 2002.

[7] T. Basar and G.J. Olsder. Dynamic Noncooperative Game Theory. Classics in Applied Mathematics, SIAM, Philadelphia, 1999.

[8] J. Bower and D. Bunn. Model-based comparisons of pool and bilateral markets for electricity. Energy Journal, 21(3):1-29, 2000.

[9] R. Byrd, M. Hribar, and J. Nocedal. An interior point algorithm for large-scale nonlinear programming. SIAM J. Optim., 9(4):877-900 (electronic), 1999. Dedicated to John E. Dennis, Jr., on his 60th birthday.

[10] A. J. Conejo, M. A. Plazas, R. Espinola, and A. B. Molina. Dayahead electricity price forecasting using the wavelet transform and arima models. IEEE Transactions on Power Systems, 20(2):1035-1042, 2005.

[11] S. P. Dirkse and M. C. Ferris. The PATH solver: A non-monotone stabilization scheme for mixed complementarity problems. CS-TR-19931179, Department of Computer Science, University of Wisconsin at Madision, 1993.

[12] R. S. Fang and A. K. David. Transmission congestion management in an electricity market. IEEE Transactions on Power Systems, 14(3):877883,1999
[13] R. Fletcher and S. Leyffer. Numerical experience with solving MPECs as NLPs, 2002.

[14] P. Giabardo and M. Zugno. Competitive Bidding and Stability Analysis in Electricity Markets Using Control Theory. Ph.D.thesis, Technical University of Denmark, Denmark, 2008.

[15] P. Giabardo, M. Zugno, P. Pinson, and H. Madsen. Feedback, competition and stochasticity in a day ahead electricity market. Energy Economics, 32:292-301, 2010.

[16] B. F. Hobbs, C. B. Metzler, and J-S. Pang. Strategic gaming analysis for electric power systems: An MPEC approach. IEEE Transactions on Power Systems, 15:638-645, 2000.

[17] R. Kamat and S. Oren. Two-settlement systems for electricity markets under network uncertainty and market power. Journal of Regulatory Economics, 25(1):5-37, 2004.

[18] S. Leyffer and T. Munson. Solving multi-leader-common-follower games. Optimization Methods Software, 25(4):601-623, 2010.

[19] S. S. Oren and A. M. Ross. Can we prevent the gaming of ramp constraints? Decis. Support Syst., 40(3-4):461-471, 2005.

[20] P. Skantze, A. Gubina, and M. Ilic. Bid-based stochastic model for electricity prices: The impact of fundamental drivers on market dynamics. Technical Report EL 00-004, Massachussets Institute of Technology, 2000.

[21] D. P. Timo and G. W. Sarney. The operation of large steam turbines to limit cyclic thermal cracking. In ASME Winter Annual Meeting and Energy Systems Exposition.

[22] D. Veit, A. Weidlich, J. Yao, and S. Oren. Simulating the dynamics in two-settlement electricity markets via an agent-based approach. International Journal of Management Science and Engineering Management, 1(2):83-97, 2006.

[23] M. Ventosa, A. Baillo, A. Ramos, and M. Rivier. Electricity market modeling trends. Energy Policy, 33:897-913, 2005.

[24] A. Wächter and L. T. Biegler. On the implementation of a primaldual interior point filter line search algorithm for large-scale nonlinear programming. Mathematical Programming, 106:25-57, 2006.

[25] C. Wang and S. M. Shahidehpour. Effects of ramp-rate limits on unit commitment and economic dispatch. IEEE Transactions on Power Systems, 8:1341-1350, 1993.

[26] J. Yao, S. Oren, and I. Adler. Computing Cournot equilibria in two settlement electricity markets with transmission constraints. In Proceeding of the 38th Hawaii International Conference on Systems Sciences (HICSS 38). Big Island, Hawaii, 2004.

The submitted manuscript has been created by the University of Chicago as Operator of Argonne National Laboratory ("Argonne") under Contract No. DEAC02-06CH11357 with the U.S. Department of Energy. The U.S. Government retains for itself, and others acting on its behalf, a paid-up, nonexclusive, irrevocable worldwide license in said article to reproduce, prepare derivative works, distribute copies to the public, and perform publicly and display publicly, by or on behalf of the Government. 